\title{
Recent SUSY results in ATLAS
}

\section{Judita Mamužić* on behalf of the ATLAS Collaboration}

Instituto de Fisica Corpuscular (IFIC) / Consejo Superior de Investigaciones Cientificas (CSIC)

- Universidad de Valencia (UV), Spain

E-mail: judita.mamuzic@cern.ch

Supersymmetry (SUSY) is considered one of the best motivated extensions of the Standard Model. It postulates a fundamental symmetry between fermions and bosons, and introduces a set of new supersymmetric particles at the electroweak scale. It addresses the hierarchy and naturalness problem, gives a solution to the gauge couplings unification, and offers a cold dark matter candidate. Different aspects of SUSY searches, using strong, electroweak, third generation production, $R$-parity violation models, and long lived particles are being studied at the LHC. An overview of most recent results in SUSY searches using Run 2 ATLAS data, at $13 \mathrm{TeV}$ with $36.1 \mathrm{fb}^{-1}$ of integrated luminosity, was presented.

Corfu Summer Institute 2017 'School and Workshops on Elementary Particle Physics and Gravity' 2-28 September 2017

Corfu, Greece

${ }^{*}$ Speaker. 


\section{Introduction}

Supersymmetry (SUSY) [1-6] is a well motivated extension of the Standard Model (SM). It postulates a fundamental symmetry between fermions and bosons, and introduces a new set of SUSY particles at the electroweak scale, where for each fermion (boson) in the SM there is a boson (fermion) SUSY partner. SUSY can offer solutions to a number of phenomena not explained by the SM. First, the radiative corrections to the Higgs boson mass become extremely large when a fermion couples to the Higgs. However, the radiative corrections of boson couplings to the Higgs are of opposite sign, and can cancel out with the fermionic contributions. SUSY naturally imposes this relation, and consequently allows large mass differences in the mass hierarchy of particles. Second, the Grand Unified Theories aim to provide one general description of electroweak and strong interactions. When experimentally measured values of electromagnetic, weak and strong coupling constants are extrapolated to high energies, these three never become equal. However, running of gauge couplings can be modified by introducing new physics between the electroweak and the Planck scale. When SUSY particles are included in the running of couplings, their evolution to the high mass scale brings to unification of gauge couplings. Third, observations of visible stars (or galactic gas) rotation speeds around the galactic center and their radial distance from it (i.e. galaxy rotation curves), show that they do not fall as the visible matter distribution prediction. They can be explained by introducing a new type of weakly interacting and non-relativistic matter, i.e. cold dark matter, and accommodate for its current estimate from astronomical measurements. SUSY offers a weakly interacting and massive particle candidate as a solution to this problem. In proton-proton collisions, SUSY particles are expected to be produced in pairs, and a typical signature has long chains of consecutive decays.

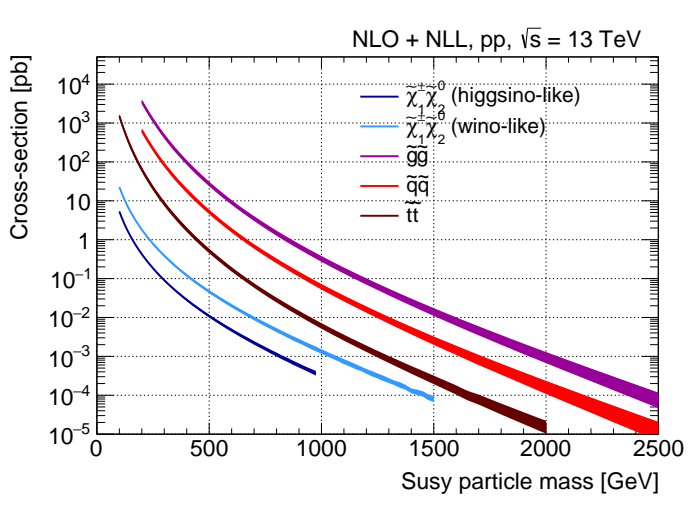

(a)

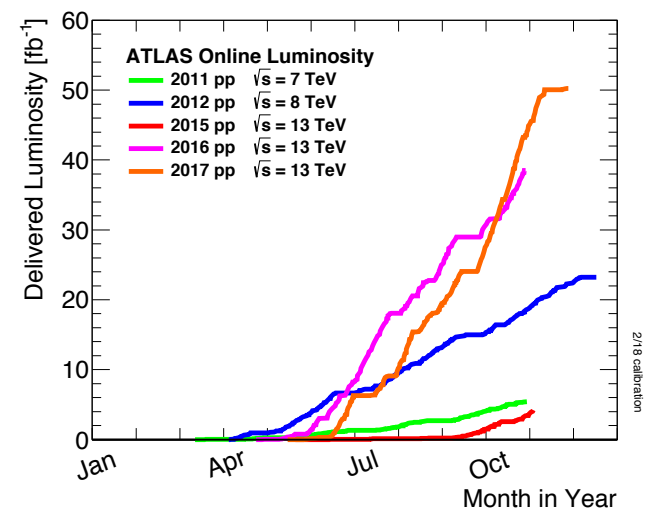

(b)

Figure 1: SUSY cross sections at $13 \mathrm{TeV}$ [7] (a) and integrated luminosity [8] collected by the ATLAS experiment (b).

SUSY searches at the LHC [9] target a broad range of final states, where each analysis defines a set of selections with high sensitivity for considered models. They are grouped around production cross sections, as shown in the Figure 1 (a). For $R$-parity conserving models these include models of $\tilde{q}$ and $\tilde{g}$ production, third generation production, and electroweak production. Also $R$-parity violating, and models with long lived particles are considered. A typical SUSY analysis defines 
a set of selections on observables. The control regions (CR) are designed to be dominated by a certain SM background, and have no or very little expected signal events. They are used to estimate the number of events in the signal regions (SR) which are designed to maximize the signalto-background ratio, while the performance of the estimate is validated in the so-called validation regions (VR), which are designed to be similar to the SRs. More details on a typical SUSY analysis in ATLAS [10] can be found in Ref. [11]. In this summary the highlights of SUSY searches are presented, using $13 \mathrm{TeV} p-p$ collision data, collected with the ATLAS experiment in 2015 and 2016 with integrated luminosity of $36.1 \mathrm{fb}^{-1}$, as shown in the Figure 1 (b).

\section{Squark and gluino production}

The production of $\tilde{q}$ and $\tilde{g}$ (strong production) has the highest cross section at the LHC, and therefore represents a very important search. High sensitivity to a large number of SUSY models can be achieved targeting a final state with jets, possible leptons, and missing transverse momentum. There are two main approaches in the searches for strong production used in ATLAS. One is the conventional, where a typical discriminating variable is the effective mass $\left(M_{\text {eff }}\right)$, defined as a scalar sum of transverse momentum of all jets, possible leptons, and missing transverse momentum. It is a good measure of all activity in the event, and is expected to have larger values for the SUSY events, compared to the SM background. The second approach is done using Recursive Jigsaw Reconstruction (RJR) variables [12]. These are kinematic variables defined on the eventby-event basis, designed to use approximations of the rest frames of the invisible (SUSY) particles in each event. They have shown to have very good sensitivity for searches with compressed SUSY mass spectrum.

The analysis with a veto on a lepton, multiple jets and missing transverse momentum [13] targets the production of $\tilde{q}$ and $\tilde{g}$. Due to a large production cross section and a very general selection, it has the sensitivity to a highest number of SUSY models. The main target of the analysis are the decays of $\tilde{q}$ and $\tilde{g}$ into quarks and $\tilde{\chi}_{1}^{0}$, the lightest symmetrical particle (LSP), and their one-step decays with intermediate $W^{ \pm}$or a $Z^{0}$ boson. A number of signal regions using the conventional and RJR analyses was designed to maximize the sensitivity to models with different $\tilde{q}, \tilde{g}$ and $\tilde{\chi}_{1}^{0}$ masses. No significant excess was seen in any of the signal regions, as shown in the Figure 2. Interpretation was done in a number of SUSY models. In Figure 3 exclusion of $\tilde{q}$ and $\tilde{g}$ pair production models, with direct and one-step decays, can be seen. For each model, a significant improvement in sensitivity of around $500 \mathrm{GeV}$ for $\tilde{q}$ and $\tilde{g}$ mass exclusion, compared to the previous analysis, using lower luminosity at $13 \mathrm{TeV} p-p$ collisions, is achieved. The $\tilde{q}$ mass is excluded up to around $1600 \mathrm{GeV}$, and $\tilde{g}$ is excluded up to around $2 \mathrm{TeV}$.

The analysis with multiple jets and missing transverse momentum targets the strong production of models with long decay chains [14]. A typical targeted model is a $\tilde{g}$ pair production with a twostep decay, where $\tilde{g}$ decays into quarks with intermediate $\tilde{\chi}_{1}^{ \pm}$, which decays into $\tilde{\chi}_{2}^{0}$ with a $W^{ \pm}$, and $\tilde{\chi}_{2}^{0}$ decays into and $Z^{0}$ and a $\tilde{\chi}_{1}^{0}$ LSP. A number of signal regions was defined, using selections with large jet multiplicity, and $b$-jet multiplicity. No significant excess was observed in any of the signal regions, and exclusion limits were set on the considered models. A large improvement in the analysis sensitivity was achieved, of around $400 \mathrm{GeV}$ for all $\tilde{g}$ masses, and in the region of 


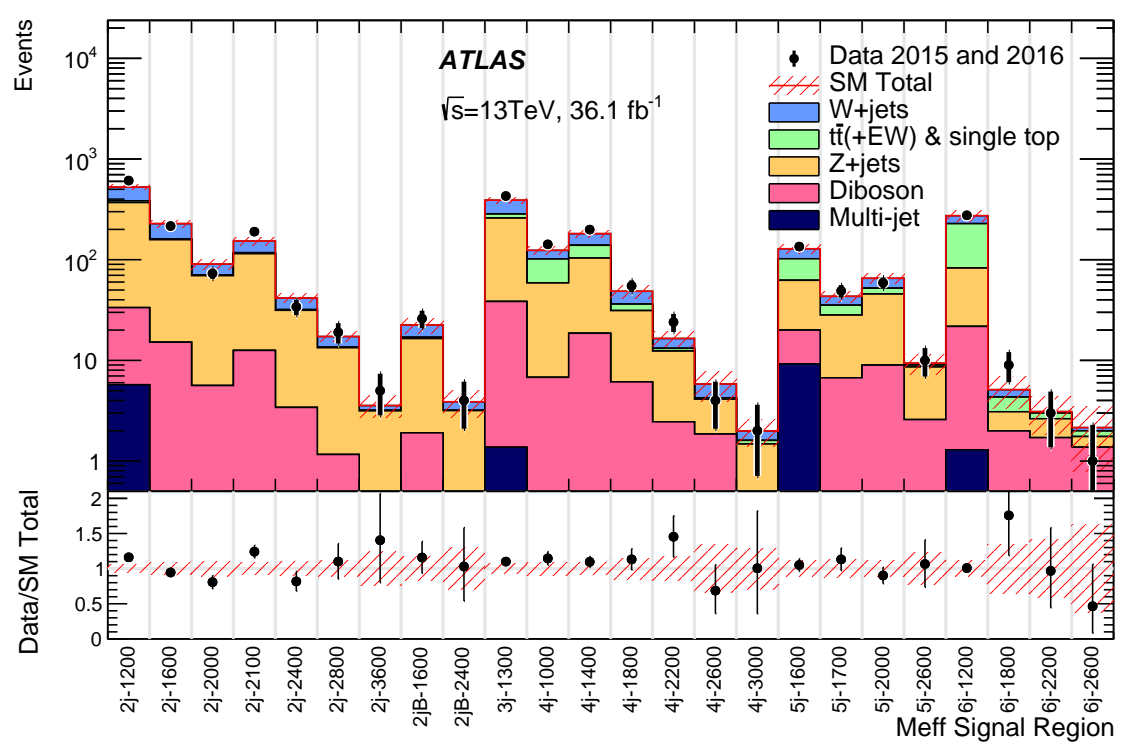

(a) $M_{\text {eff }}$

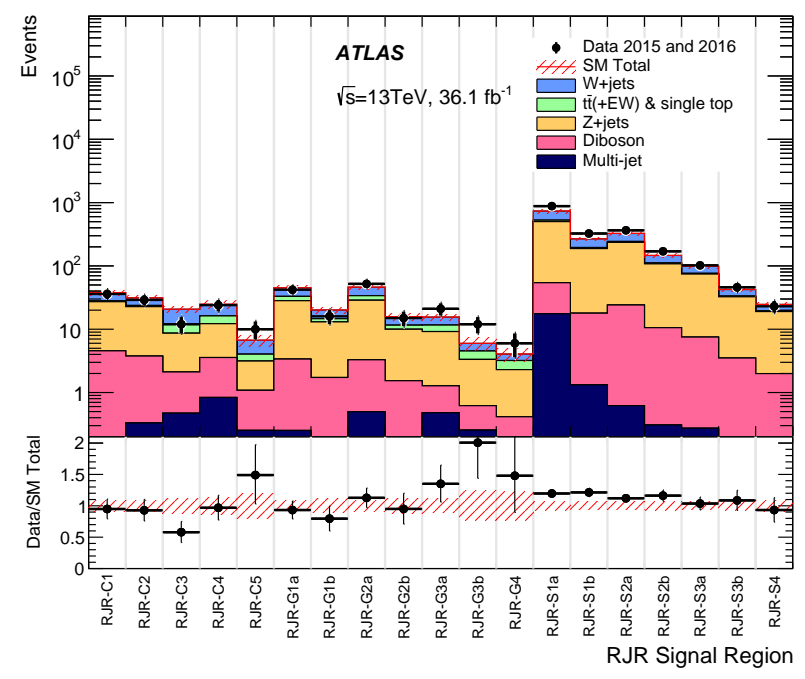

(b) RJR

Figure 2: Signal regions for the SUSY analysis with a veto on a lepton, multiple jets and missing transverse momentum using conventional (a) and RJR (b) variables [13].

compressed mass spectrum in the diagonal of the $\tilde{g}$ vs $\tilde{\chi}_{1}^{0}$ plane, compared to the previous $13 \mathrm{TeV}$ search. Gluino masses up to around $1800 \mathrm{GeV}$ were excluded, as shown in the Figure 4.

A distinctive signature of SUSY is with at least two same-sign leptons, and this analysis is targeted with a search with two same-sign or three leptons [15]. Signal regions were optimized for a number of $R$-parity conserving and $R$-parity violating models, which differ in the selection on a number of leptons, same-sign requirement and $b$-jet multiplicity. No significant excess was observed in any of the signal regions, and exclusion limits were set on a number of SUSY models. In the exclusion of the $\tilde{g}$ pair production with a two-step decay, a large improvement in sensitivity for 

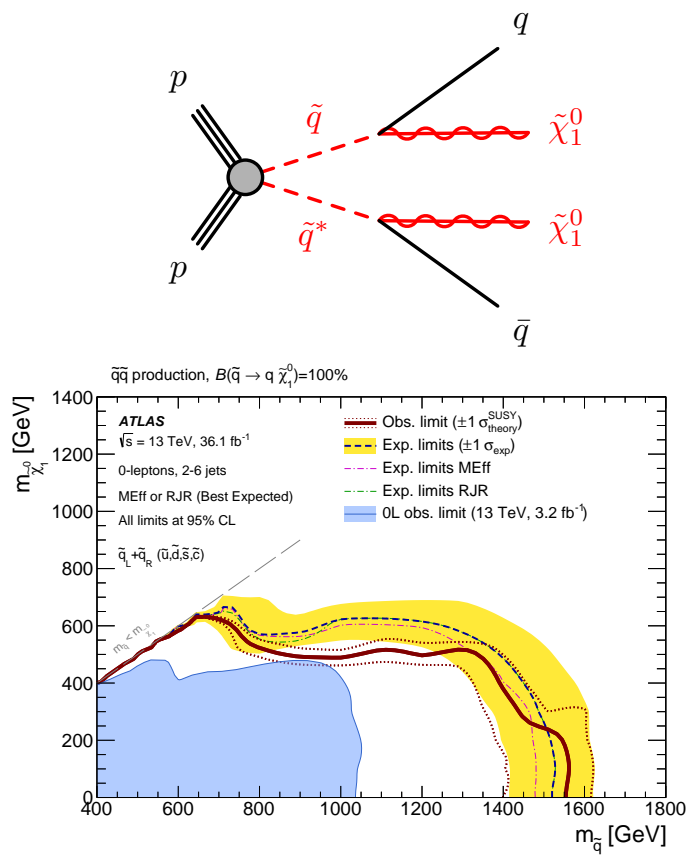

(a) $\tilde{q} \tilde{q}$ production, direct decay
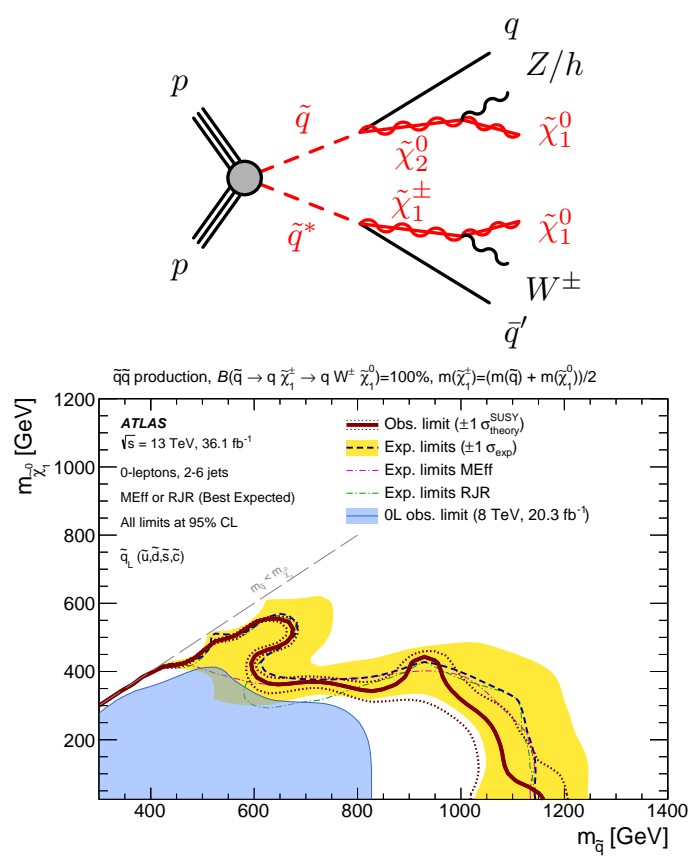

(c) $\tilde{q} \tilde{q}$ production, one-step decay
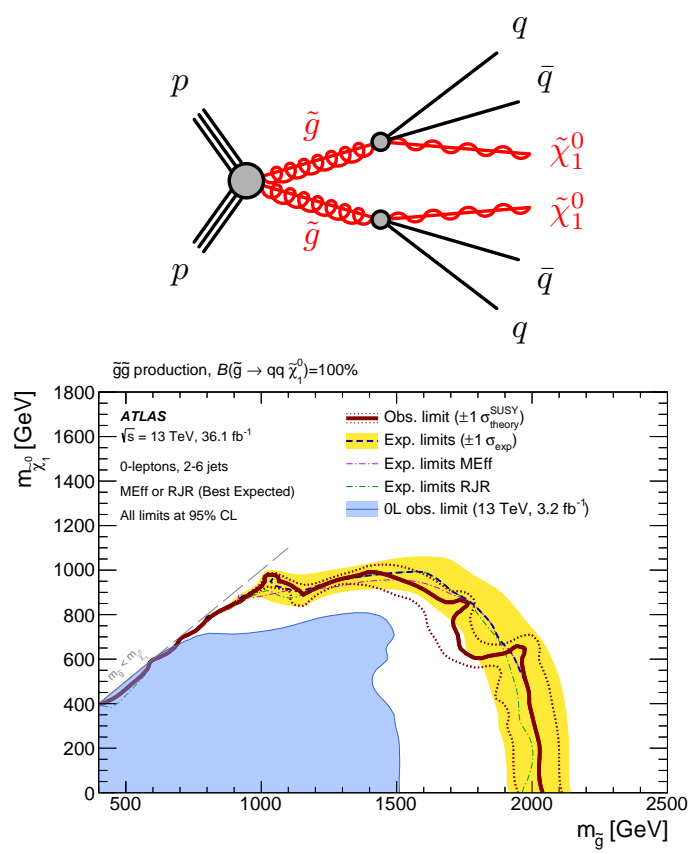

(b) $\tilde{g} \tilde{g}$ production, direct decay
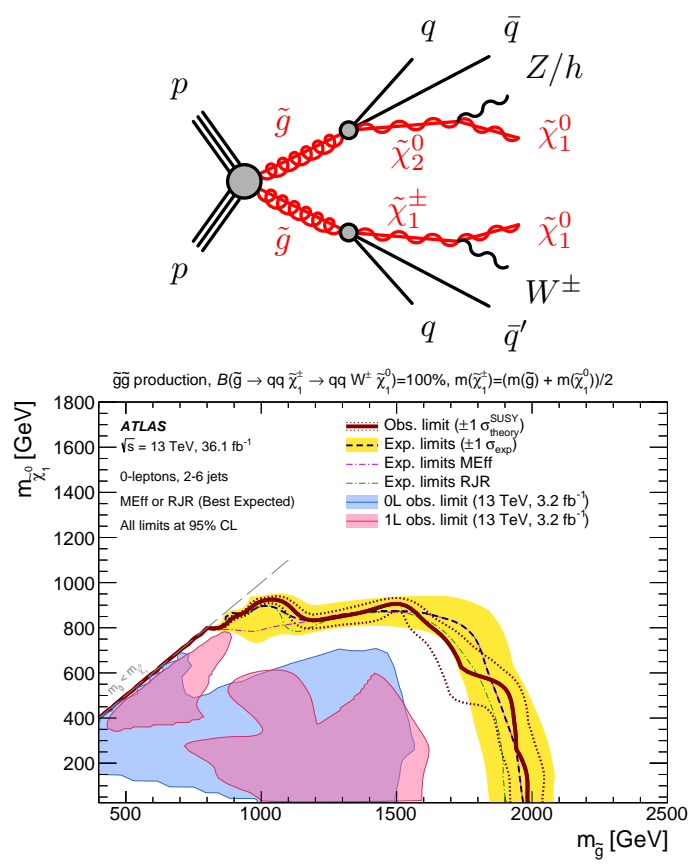

(d) $\tilde{g} \tilde{g}$ production, one-step decay

Figure 3: Interpretation of simplified $\tilde{q}$ (left) and $\tilde{g}$ (right) production models, with direct (up) and one-step decays (down) using the analysis with a veto on a lepton, multiple jets and missing transverse momentum [13]. 

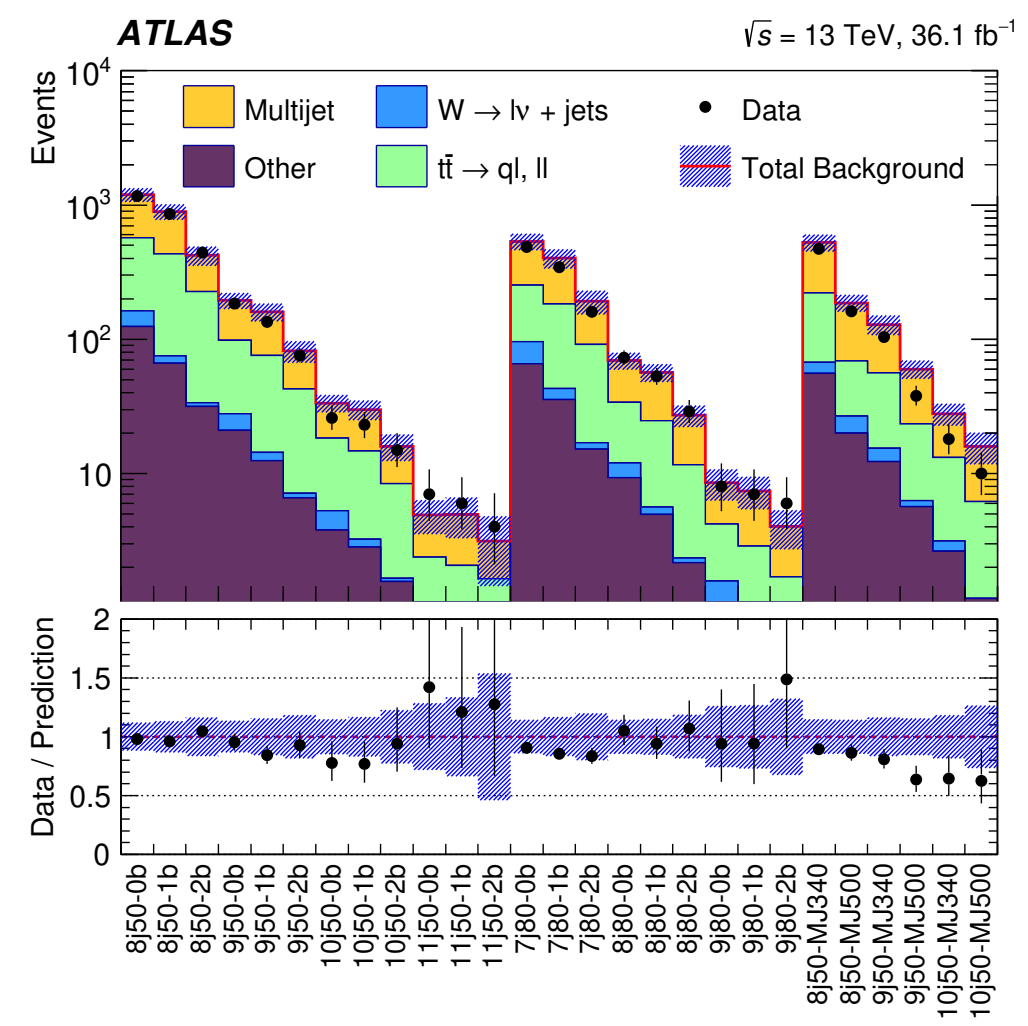

(a) SRs

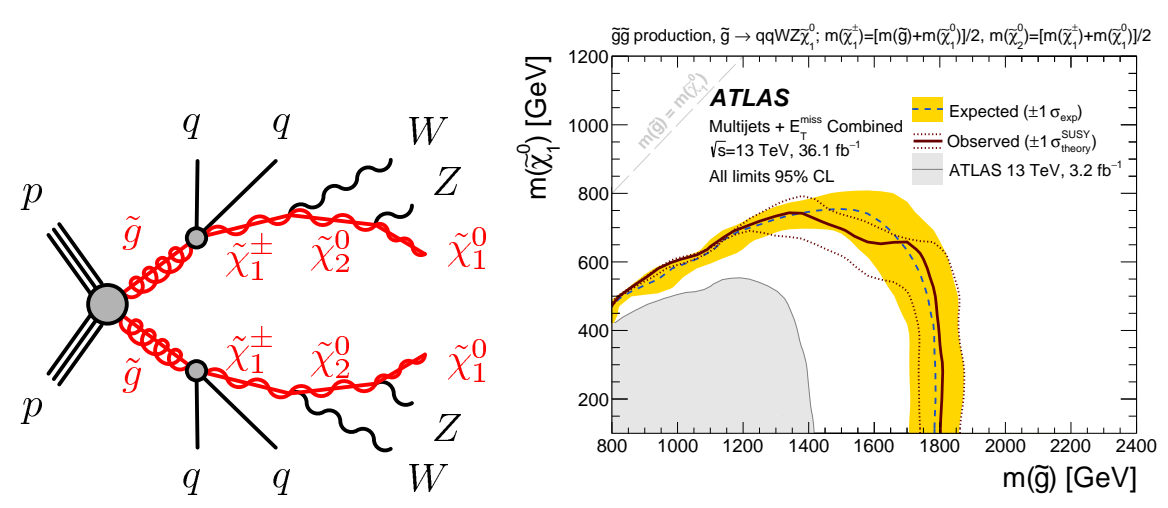

(b) $\tilde{g} \tilde{g}$ two-step decay

Figure 4: Analysis with multiple jets and missing transverse momentum [14]. No significant excess observed in any of the signal regions (a), and exclusion limits for the $\tilde{g}$ pair production with a two-step decay (b). 
the $\tilde{g}$ masses of about $500 \mathrm{GeV}$ was achieved, and in the region with compressed mass spectrum. Gluino masses up to around $1600 \mathrm{GeV}$ were excluded, as shown in the Figure 5.

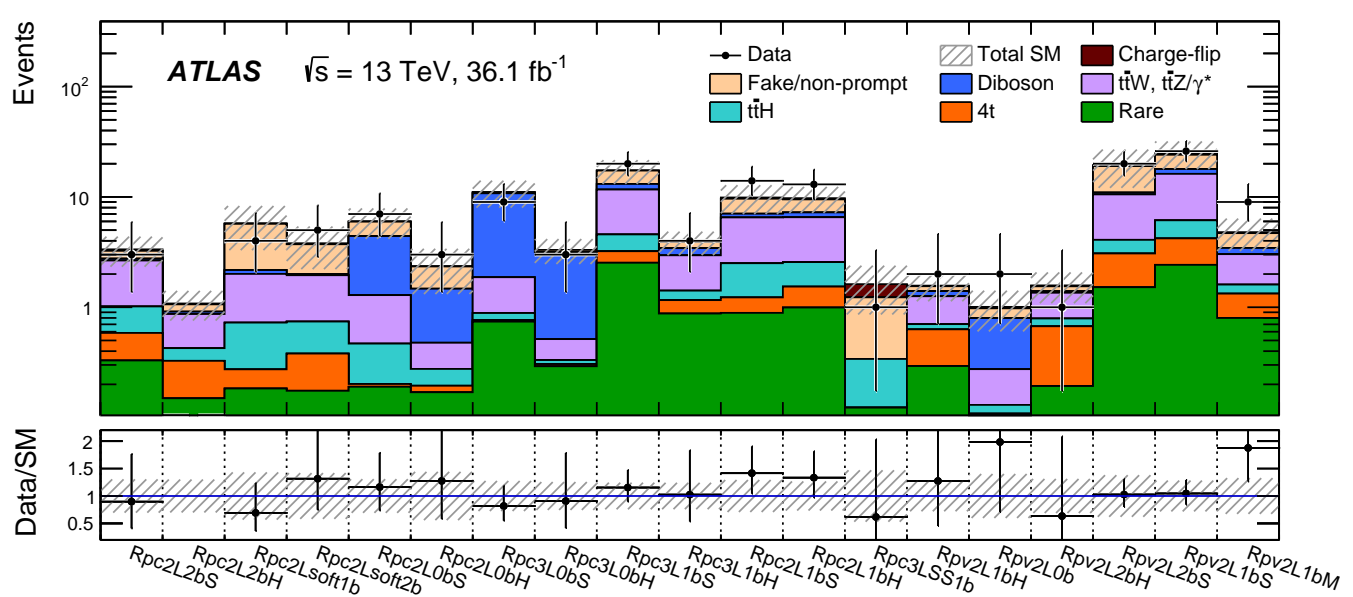

(a) SRs
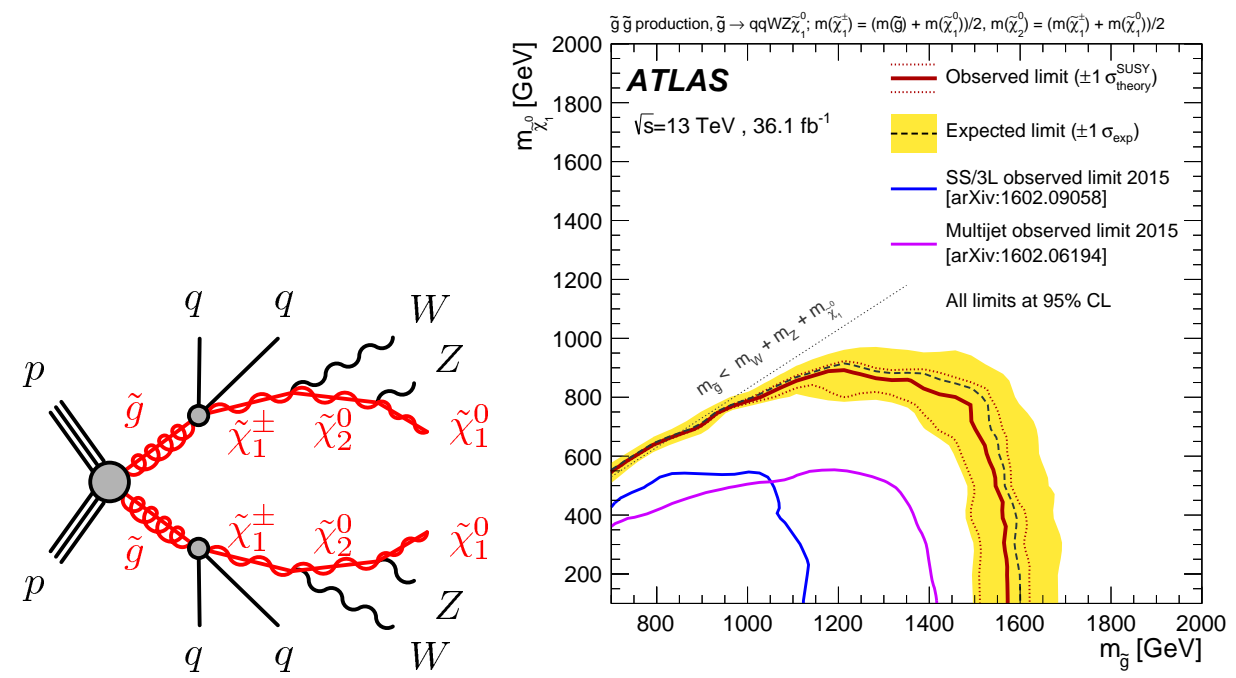

(b) $\tilde{g} \tilde{g}$ two-step decay

Figure 5: Analysis with two same-sign or three leptons [15]. No significant excess in any of the signal regions seen (a), and exclusion limits for the $\tilde{g}$ pair production with a two-step decay (b).

In addition to searches with light $(u$ and $d$ ) quarks, an analysis of SUSY models with decays of a $\tilde{g}$ with third generation quarks ( $b$ and $t$ ) [16] needs to be considered. The signal regions were optimized for models with direct and one-step decays of $\tilde{g}$ with $b$ and $t$ quarks. No significant excess was observed in any of the signal regions, and exclusion limits were set. Gluino masses up to about $1.8-2 \mathrm{TeV}$ were excluded, as shown in the Figure 6.

In the Figure 7 a comparison of strong production searches is shown. Highest exclusion in the $\tilde{g}$ masses of around $2 \mathrm{TeV}$ is reached by the analysis with no leptons, 2-6 jets and missing transverse momentum, and it is comparable to the reach of the gluino mediated third generation searches, which in addition have a higher coverage toward the higher $\tilde{\chi}_{1}^{0}$ masses. 


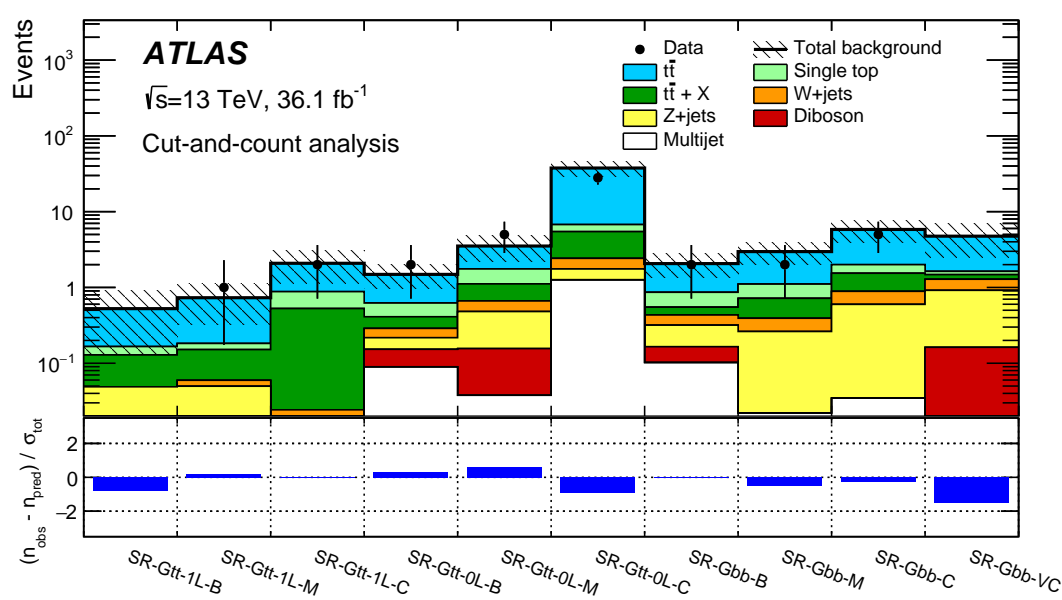

(a) SRs
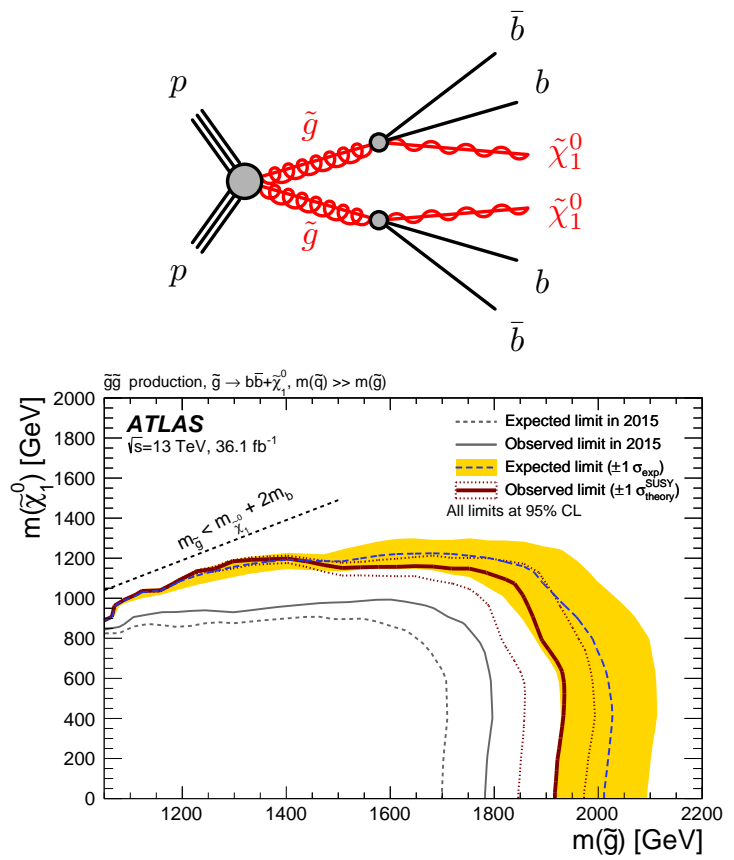

(b) $\tilde{g} \tilde{g}$ to bb direct decay
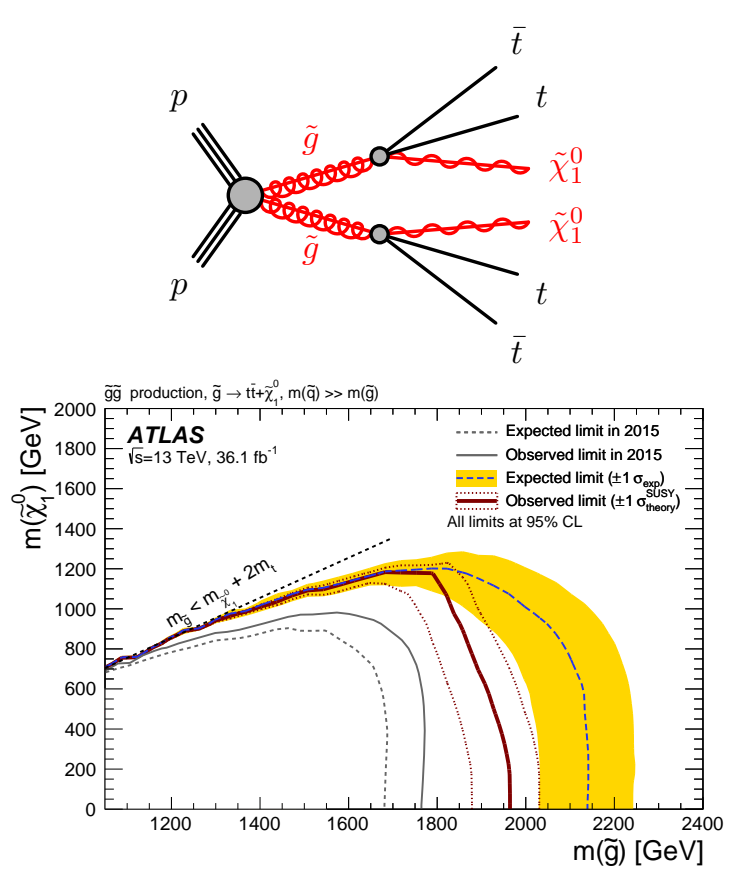

(c) $\tilde{g} \tilde{g}$ to tt direct decay

Figure 6: Gluino mediated third generation search [16]. No significant excess in any of the signal regions, exclusion limits for direct gluino decays with $b$ and $t$ quarks (b). 


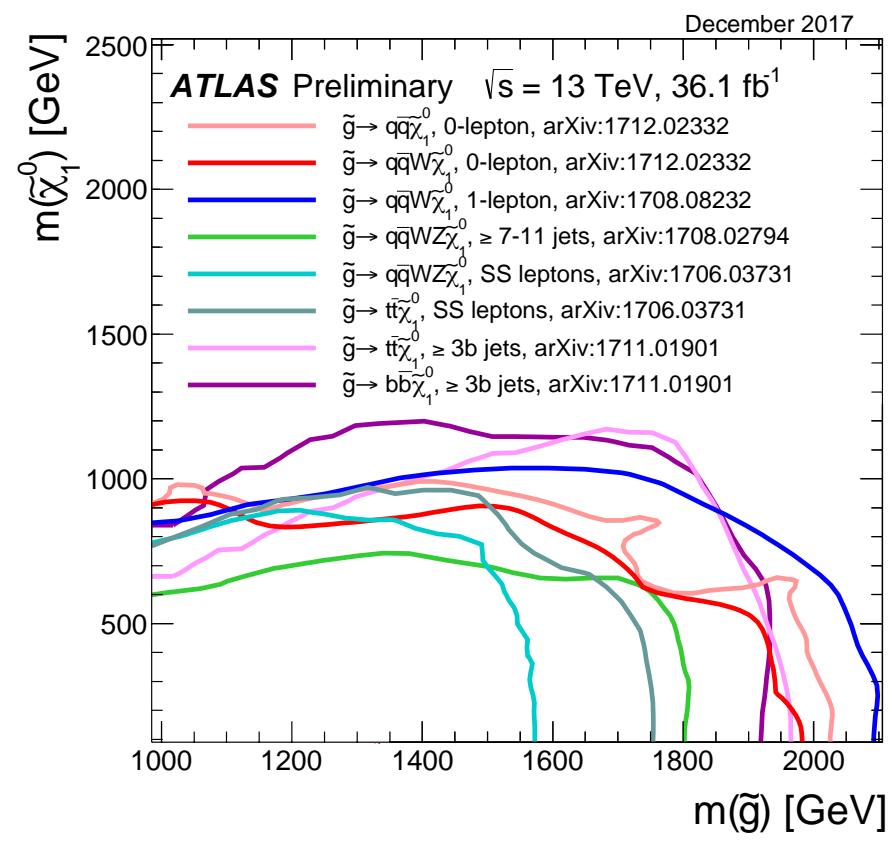

Figure 7: Strong production analyses summary [17]. Exclusion curves shown for different $\tilde{g}$ decays.

\section{Third generation production}

A strong motivation for third generation searches is given by the Higgs mass measurement $[18,19]$, which seems to be unnaturally light, and the existing $\tilde{q}$ and $\tilde{g}$ exclusion limits which reach up to the $\mathrm{TeV}$ scale. The dominant contribution to the Higgs mass comes from the divergent term of the top-quark. If SUSY exists, and $\tilde{t}$ has masses $\leq 1 \mathrm{TeV}$, loop diagrams of the top-quark can cancel out to a large extent, which gives a natural solution to the mass hierarchy problem. In addition, large splitting between $\tilde{t}_{1}$ and $\tilde{t}_{2}$ can be achieved due to large $t$ Yukawa couplings, so effects of the renormalization group equations are high for the third generation squarks, which brings to low $\tilde{t}_{1}$ masses compared to first generation $\tilde{q}$ masses. In this light, a dedicated search for light $\tilde{t}$ is well motivated.

In order to obtain good sensitivity for a variety of typical signatures of $\tilde{t}$ decays, in optimization a simplified model approach was used, where $\tilde{\chi}_{1}^{0}$ LSP was assumed to be bino-like [20], see more details in the Figure 8. For mass differences of $\Delta m=m_{\tilde{t}_{1}}-m_{\tilde{\chi}_{1}^{0}}$ higher than the stop mass $m_{\tilde{t}_{1}}$, a $\tilde{t}$ pair production with the direct decays of $\tilde{t}$ with a $t$ quark was used in optimization. Similarly, one-step decays with $b$ quarks and intermediate $\tilde{\chi}_{1}^{ \pm}$decaying into a $W^{ \pm}$and $\tilde{\chi}_{1}^{0}$ LSP, were considered for the $\Delta m>m_{W^{ \pm}}+m_{b}$. Further dedicated selections were optimized for even lower $\Delta m$, where the $W^{ \pm}$decays off-shell, and for $\tilde{t}$ decaying into a $c$ quark and $\tilde{\chi}_{1}^{0}$ LSP. No significant excess was seen in any of the signal regions, and exclusion limits were set. A large improvement in sensitivity, of about $250 \mathrm{GeV}$ in the $\tilde{t}$ mass for the low $\tilde{\chi}_{1}^{0}$, and strongly improved coverage for the lower $\Delta m$ models, was achieved compared to $13 \mathrm{TeV}$ searches with lower integrated luminosity.

In addition to using simplified models with a bino $\tilde{\chi}_{1}^{0}$ LSP, more realistic mass spectrum 


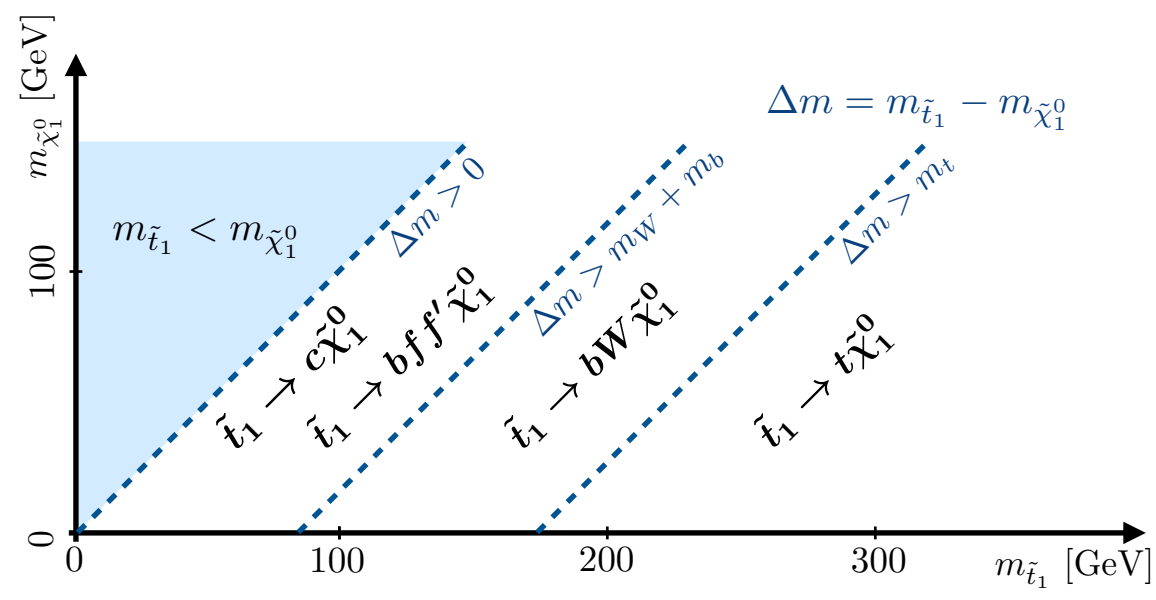

(a) Analysis strategy

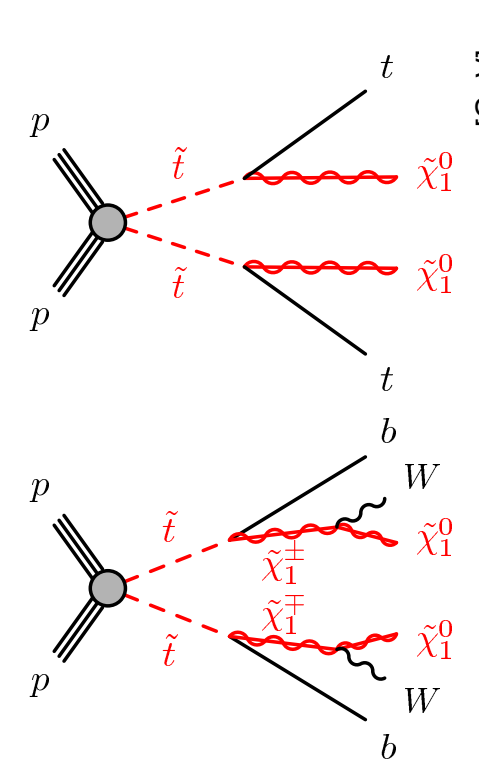

(b) $\tilde{t} \tilde{t}$ direct and one step decay

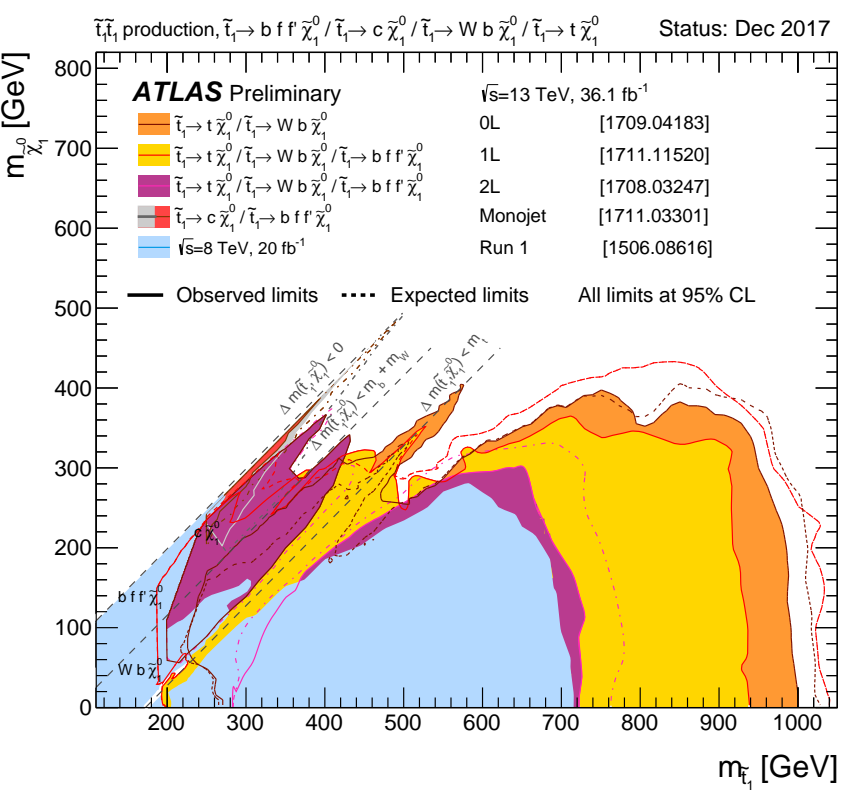

(c) $\tilde{t}$ analysis summary [17].

Figure 8: $\tilde{t}$ pair production analysis strategy using simplified models [20] (a) and exclusion limits [17] (b).

and other LSP mixtures need to be considered for $\tilde{t}$ pair production. For this, dedicated model specifications in Phenomenological Minimal SUSY Model (pMSSM) [22,23] were used, as shown in the Figure 9. Models motivated by gauge unification at the GUT scale predict a wino $\tilde{\chi}_{1}^{0}$ LSP, and have $\tilde{\chi}_{1}^{ \pm}$and $\tilde{\chi}_{2}^{0}$ masses in between third generation squarks, and $\tilde{\chi}_{1}^{0}$ LSP. Models motivated by obtaining natural SUSY, have a Higgsino LSP, and favor light $\tilde{\chi}_{1}^{ \pm}, \tilde{\chi}_{2}^{0}$ and $\tilde{\chi}_{1}^{0}$ LSP, with a large mass difference compared to $\tilde{t}_{1}$. Models that predict a dark matter candidate produced in the right amount favor a bino-Higgsino mixture, where third generation squarks have a higher mass compared to all neutralinos and charginos, with $\tilde{\chi}_{1}^{0}$ even slightly lighter. Dedicated analyses were optimized to target these scenarios. No significant excess was seen in any of the searches, and 


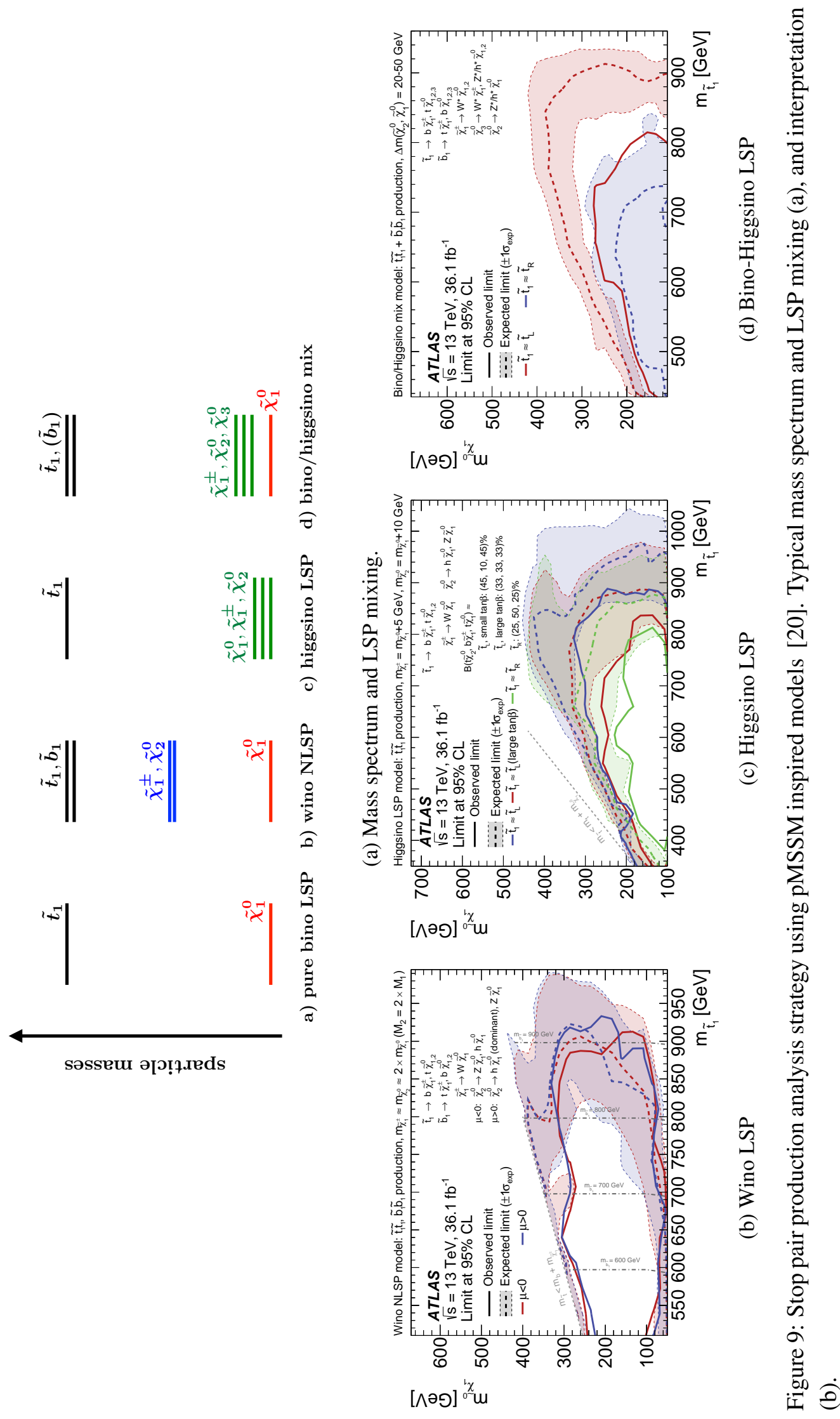




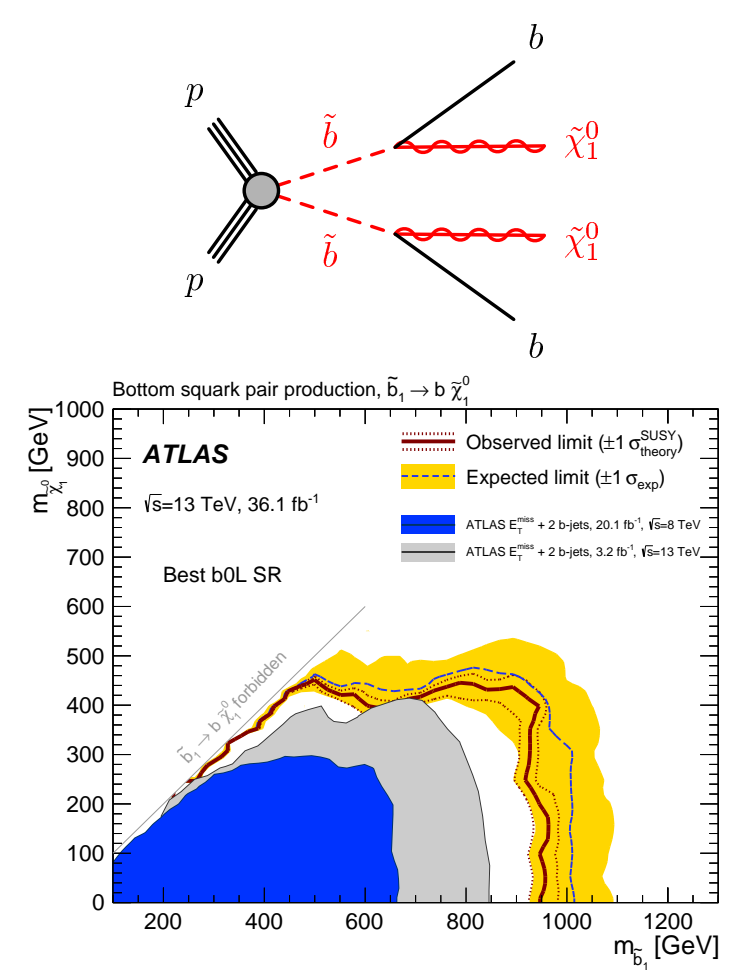

(a) $\tilde{b} \tilde{b}$ direct decay
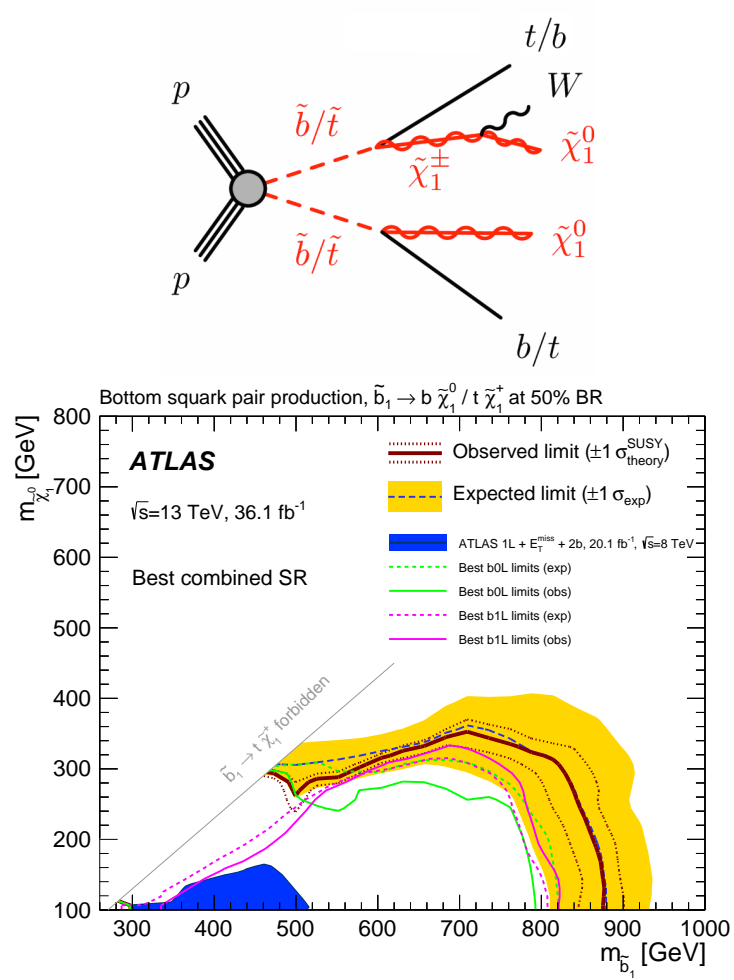

(b) $\tilde{b} \tilde{b}$ one-step decay

Figure 10: Search for $\tilde{b}$ pair production [21].

exclusion limits were set on these models [20]. These are the first exclusions of pMSSM inspired models in ATLAS, dedicated for $\tilde{t}$ pair production, with these given motivations.

In addition to $\tilde{t}$ pair production, a complementary search is done using the $\tilde{b}$ quark production [21]. Typical final states in the analysis include a $\tilde{b}$ pair production, with a direct decay into a $b$ quark and $\tilde{\chi}_{1}^{0}$ LSP, or $\tilde{b}$ one-step decay modes via $\tilde{\chi}_{1}^{ \pm}$with a $W^{ \pm}$in the decay chain. For the direct production, an improvement in the sensitivity of about $100 \mathrm{GeV}$ in the $\tilde{b}$ mass is achieved, while for the combined direct and one-step decays in the $\tilde{b}$ pair production an impressive $400 \mathrm{GeV}$ improvement is achieved for the $\tilde{b}$ mass exclusion, and significant improvement in sensitivity is achieved for the compressed mass spectrum towards the diagonal in the $\tilde{b}$ vs $\tilde{\chi}_{1}^{0}$ plane, compared to the previous searches. The exclusion limits of $\tilde{b}$ reach about $900-1000 \mathrm{GeV}$ for direct and combined decays, as shown in Figure 10.

\section{Electroweak production}

If the masses of squarks and gluinos are significantly large, the production of $\tilde{\chi}^{0}, \tilde{\chi}_{1}^{ \pm}$and $\tilde{\ell}$ can be dominant. As the limits of the strong production are reaching about $2 \mathrm{TeV}$, the studies of the electroweak production become well motivated. Typical searches consider final states with two or three leptons, with and without jets, in the $\tilde{\chi}_{1}^{ \pm}$pair, $\tilde{\ell}$ pair, and $\tilde{\chi}_{2}^{0} \tilde{\chi}_{1}^{ \pm}$production [24]. Significant improvement in the sensitivity to considered models was obtained, compared to previous searches, by using the analyses with multiple bin fits. The analyses with $\tilde{\chi}_{2}^{0} \tilde{\chi}_{1}^{ \pm}$production, with $W^{ \pm}$and 
$Z^{0}$ in the decay chains were using the binned invariant mass of two leptons. Models with $\tilde{\ell}$ in the decay chain were using the binned information of the $m_{T 2}$ variable, which is a good measure of the missing transverse momentum in-balance in the event. No significant excess was observed in any of the signal regions, and exclusion limits were set, as shown in the Figure 11.

A comparison of recent searches using the electroweak production [17] is shown in Figure 12. A number of final states for the $\tilde{\ell}$ and $\tilde{\chi}_{2}^{0} \tilde{\chi}_{1}^{ \pm}$production is targeted. The highest exclusion limits were set for the $\tilde{\chi}_{2}^{0} \tilde{\chi}_{1}^{ \pm}$production, with decays via $\tilde{\ell}$ or $\tilde{v}$ up to $1.16 \mathrm{TeV}$ for $\tilde{\chi}_{2}^{0} / \tilde{\chi}_{1}^{ \pm}$masses.

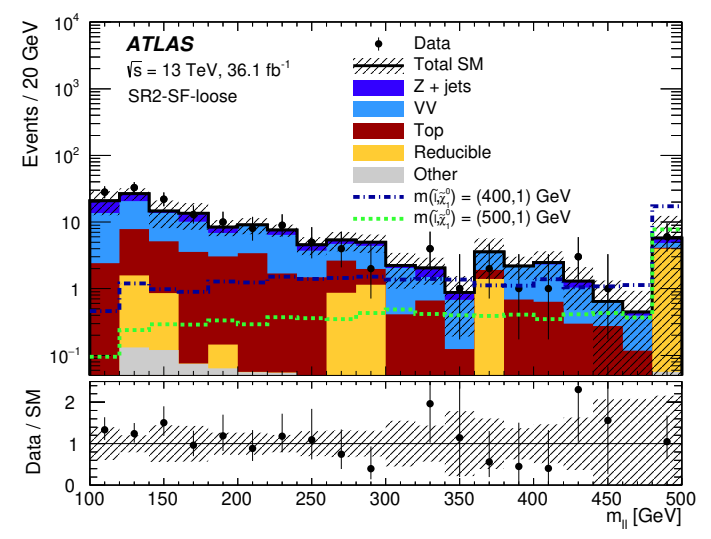

(a) $\mathrm{m}_{l l}$
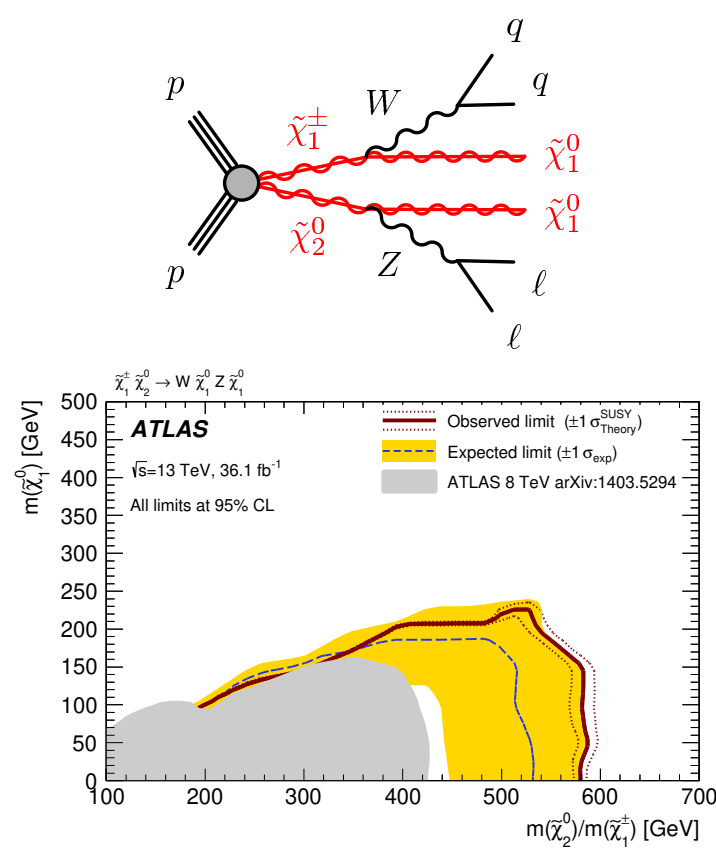

(c) $\tilde{\chi}_{2}^{0} \tilde{\chi}_{1}^{0}$ with $W^{ \pm} Z^{0}$

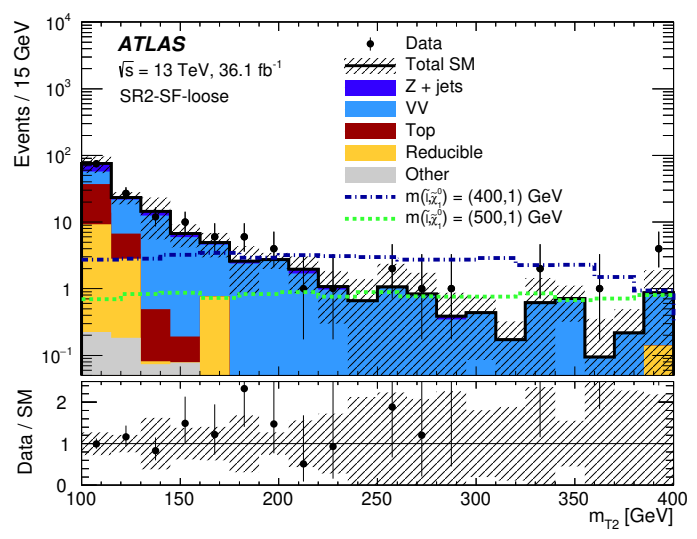

(b) $\mathrm{m}_{T 2}$
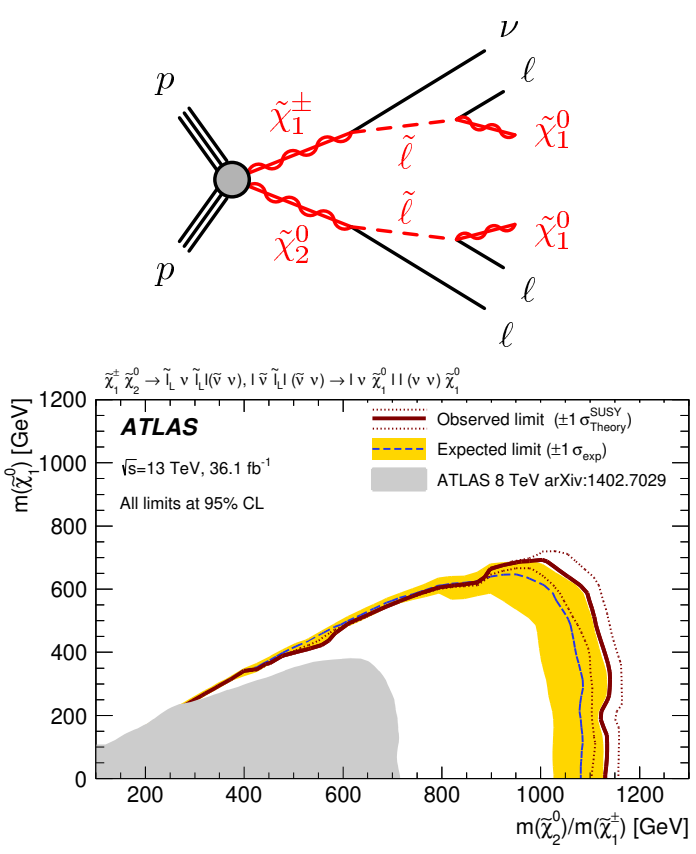

(d) $\tilde{\chi}_{2}^{0} \tilde{\chi}_{1}^{0}$ with $\tilde{\ell}$

Figure 11: Search for electroweak production using two or three leptons [24], using multibin fit for in $m_{l l}$ (left) and $m_{T 2}$ (right) and interpretation (bottom). 


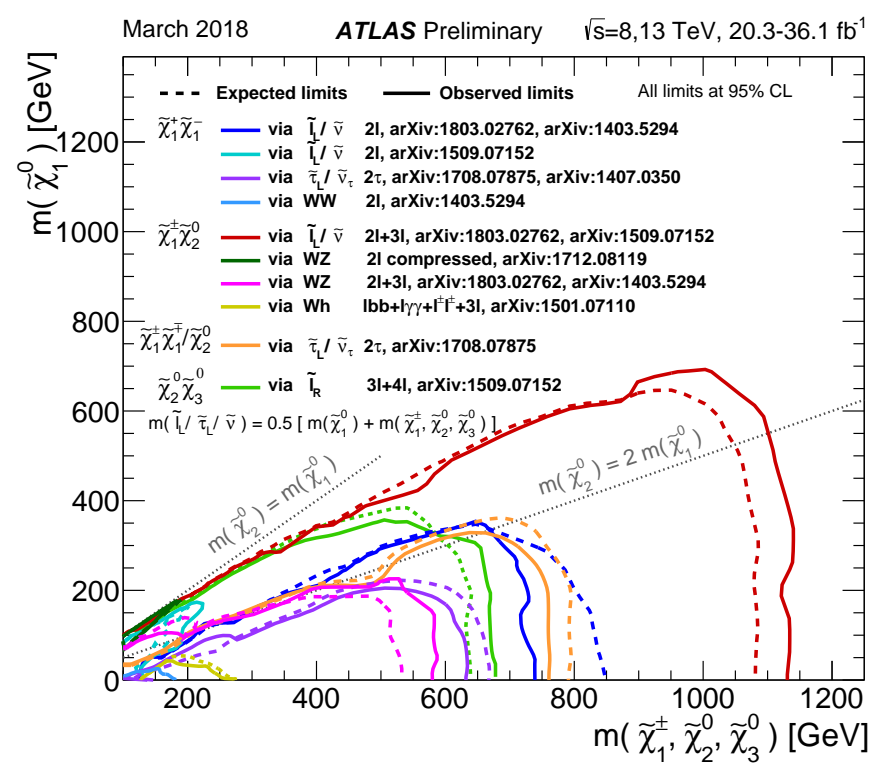

Figure 12: Electroweak production analyses summary [17]. Exclusion curves shown for different productions and decays.

\section{Long lived particles}

The searches for SUSY models where sparticles are long lived, require dedicated analyses. The search for the the long lived $\tilde{\chi}_{1}^{ \pm}$is targeted using the so-called disappearing-track analysis [17]. The production with long lived $\tilde{\chi}_{1}^{ \pm}, \tilde{\chi}_{1}^{0}$ and initial-state-radiation jet, where $\tilde{\chi}_{1}^{ \pm}$decays into a pion and $\tilde{\chi}_{1}^{0}$ LSP, and similarly, a $\tilde{g}$ pair production, where $\tilde{g}$ can decay into two quarks and $\tilde{\chi}_{1}^{0}$ LSP or into two quarks and long lived $\tilde{\chi}_{1}^{ \pm}$, which decays into a pion and $\tilde{\chi}_{1}^{0}$ LSP, were considered. The long lived $\tilde{\chi}_{1}^{ \pm}$leaves hits in the inner parts of the detector (Pixel), and decays into a pion and $\tilde{\chi}_{1}^{0}$ LSP, and has no counterparts of the track in the further parts of the tracking detector (SCT), as shown in the Figure 13 (a). The improved sensitivity of the analysis makes use of the implemented additional layer in the Pixel detector, and improves the sensitivity towards shorter life times of the long lived $\tilde{\chi}_{1}^{ \pm}$. No significant excess was observed in any of the signal regions, and a comparison to previous searches [17] is shown in the Figure 13 (b).

\section{6. $R$-parity violation}

In addition to $R$-parity conserving models, the searches for $R$-parity violating models (RPV) need to be considered. Due to $R$-parity violating terms in the SUSY hyperpotential, in these models lepton and baryon number violation are allowed. This brings to a variety of final states not considered in standard searches, and dedicated analyses were set in place. A broad range of new RPV searches were considered compared to analyses with lower luminosity, but no significant excess was observed in any of the dedicated signal regions [26]. Exclusion limits were set, and for gluino pair production, with $\tilde{g}$ decaying into a $t$ and $\tilde{t}$, where $\tilde{t}$ decays into a $b$ and $s$ quark, $\tilde{g}$ is excluded up to masses of about $1600 \mathrm{GeV}$. Similarly, for a $\tilde{g}$ pair production, with a decay into a 


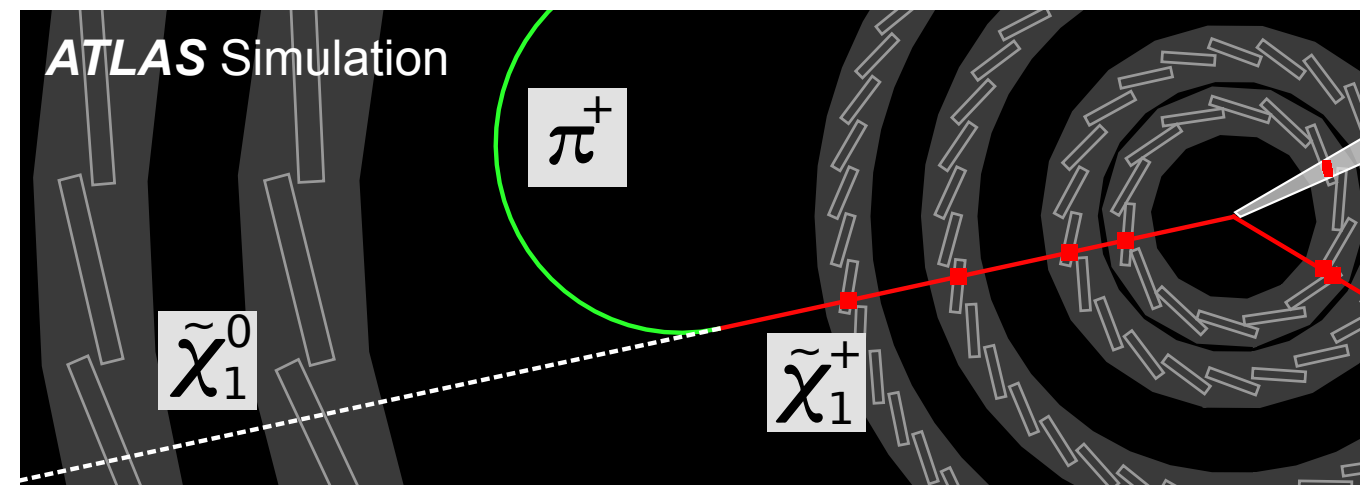

(a) "Disappearing track" analysis strategy.

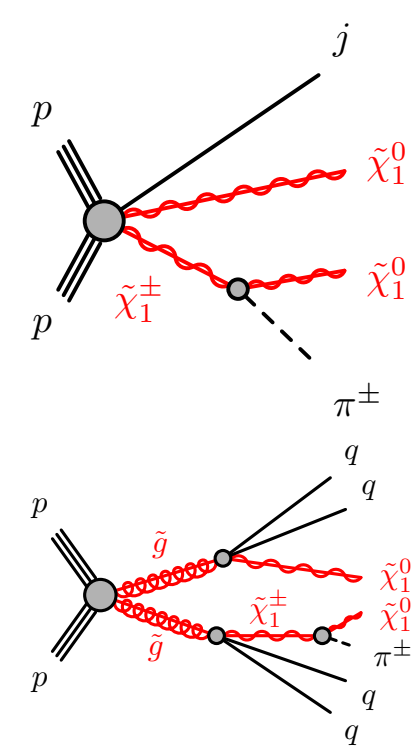

(b) Long lived $\tilde{\chi}_{1}^{ \pm}$

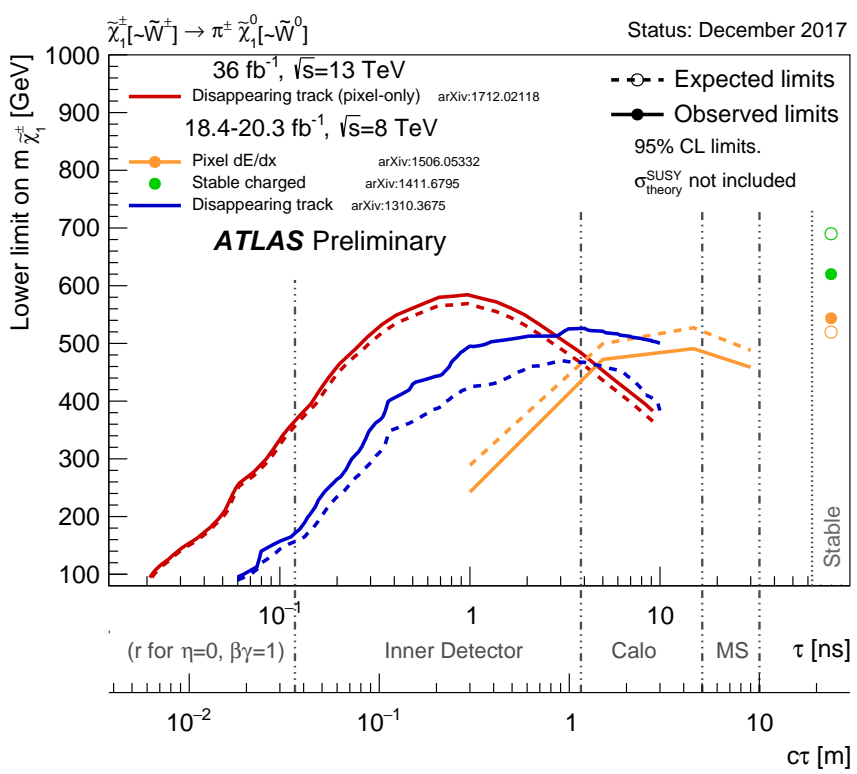

(c) Long lived $\tilde{\chi}_{1}^{ \pm}$summary [17].

Figure 13: Long lived $\tilde{\chi}_{1}^{ \pm}$analysis strategy (a) [17] and excluion summary (b) [25].

pair of $t$ quarks and intermediate $\tilde{\chi}_{1}^{ \pm}$or $\tilde{\chi}_{1}^{0}$, where both of these sparticles decay into three quarks, $\tilde{g}$ masses are excluded up to about $2 \mathrm{TeV}$, as shown in the Figure 14.

\section{Conclusion}

A broad range of improvements were implemented for the SUSY analyses using the $36.1 \mathrm{fb}^{-1}$ of ATLAS data. No significant excess in any of the signal regions was observed, and exclusion limits were set. In the $\tilde{q}$ and $\tilde{g}$ production, optimization for different regions of model parameter space brings to large improvements in the sensitivity of $\tilde{g}$ and $\tilde{q}$ masses, compared to searches with lower luminosity. In the searches for the third generation production, impressive improvements were obtained in the sensitivity of the analyses for standard and compressed mass regions. In addition, a new type of models were considered using the pMSSM parameter space selection, targeting well motivated LSP mixture scenarios. In the electroweak production, higher sensitivity was obtained 

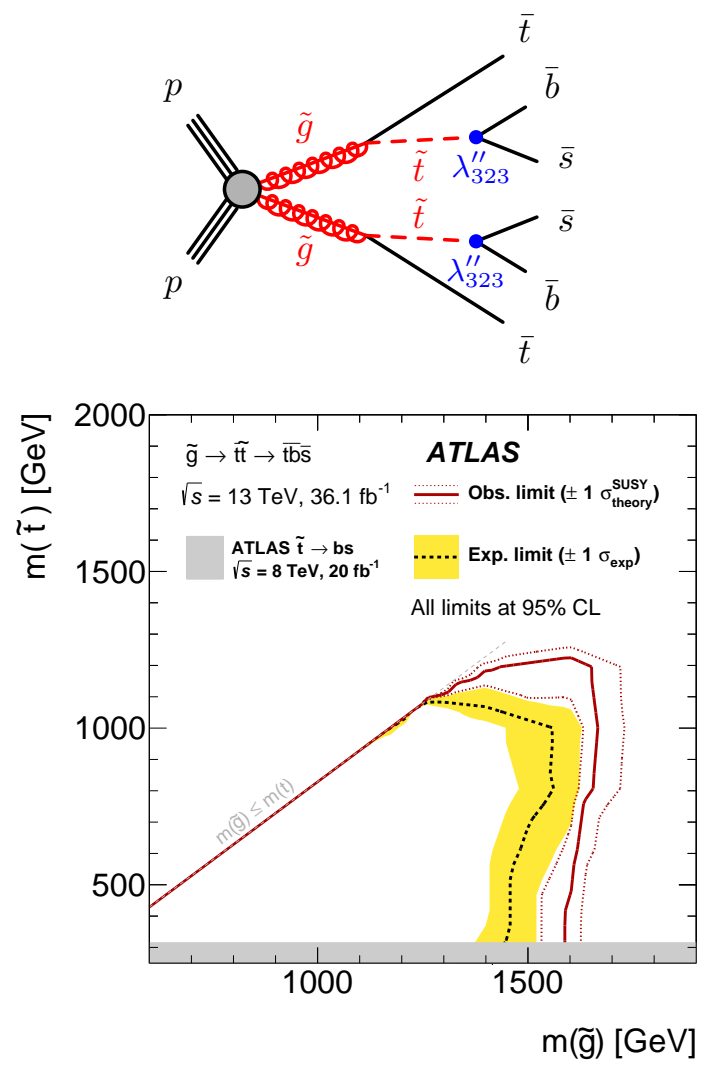

(a) RPV $\tilde{g} \tilde{g}$ direct decay
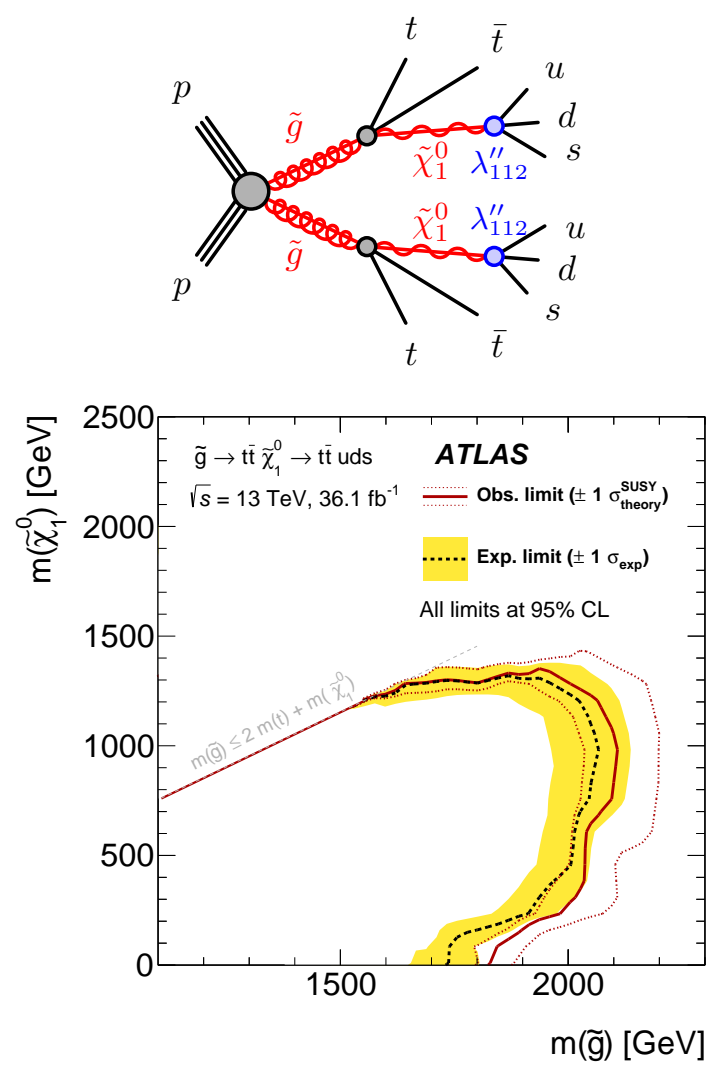

(b) RPV $\tilde{g} \tilde{g}$ one-step decay

Figure 14: $R$-parity violating models interpretation [26], $\tilde{g}$ pair production with $\tilde{t}$ decaying into two quarks (left), and $\tilde{g}$ pair production with $\tilde{\chi}_{1}^{0}$ and $\tilde{\chi}_{1}^{ \pm}$decaying to three quarks (right).

using improved searches and higher luminosity. For the long lived particles, limits on the long lived $\tilde{\chi}_{1}^{ \pm}$were set, which benefited from the newly added Pixel detector layer. In addition to $R$-parity conserving models, limits were set on a broad range of $R$-parity violating models. In the Figure 15 a summary of recent SUSY searches in ATLAS is shown [17]. Future searches in ATLAS are planned to use more than two times higher luminosity, and a large number of improvements are set in place. On the one side in extending the sensitivity for uncovered and difficult parameterspace of existing searches, and on the other side in improving the searches by using a large number of new well motivated physics scenarios.

\section{Acknowledgements}

The author acknowledges support by the Spanish MINEICO under the project FPA201565652-C4-1-R and by the Severo Ochoa Excellence Centre Project SEV-2014-0398. 


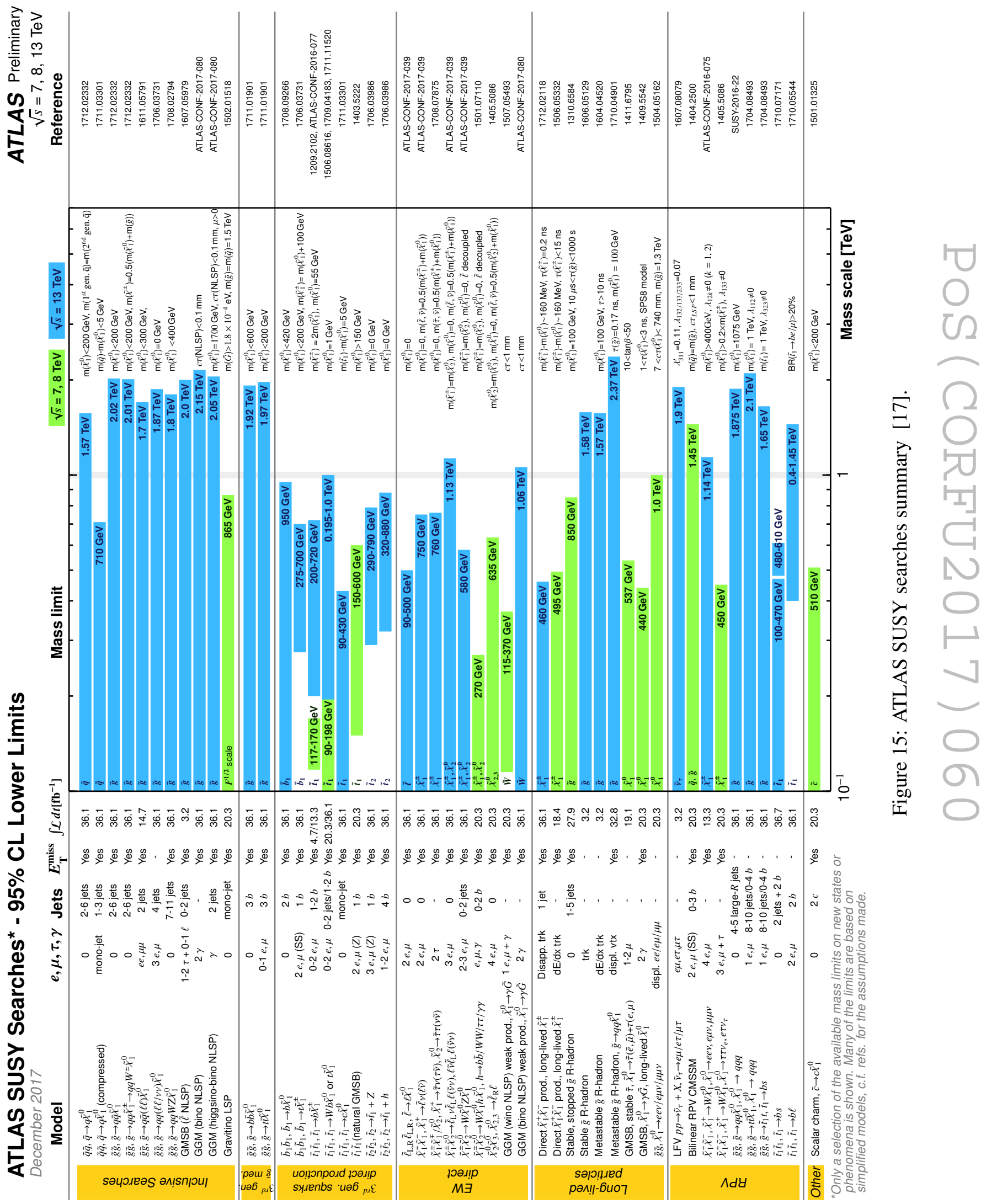




\section{References}

[1] Yu. A. Golfand and E. P. Likhtman. Extension of the Algebra of Poincare Group Generators and Violation of p Invariance. JETP Lett., 13:323, 1971. [Pisma Zh. Eksp. Teor. Fiz. 13 (1971) 452]. URL: http: //inspirehep.net/record/68412.

[2] D. V. Volkov and V. P. Akulov. Is the Neutrino a Goldstone Particle? Phys. Lett. B, 46:109, 1973. doi:10.1016/0370-2693(73)90490-5.

[3] J. Wess and B. Zumino. Supergauge Transformations in Four-Dimensions. Nucl. Phys. B, 70:39, 1974. doi:10.1016/0550-3213(74) 90355-1.

[4] J. Wess and B. Zumino. Supergauge Invariant Extension of Quantum Electrodynamics. Nucl. Phys. B, 78:1, 1974. doi:10.1016/0550-3213(74)90112-6.

[5] S. Ferrara and B. Zumino. Supergauge Invariant Yang-Mills Theories. Nucl. Phys. B, 79:413, 1974. doi:10.1016/0550-3213(74)90559-8.

[6] Abdus Salam and J. A. Strathdee. Supersymmetry and Nonabelian Gauges. Phys. Lett. B, 51:353, 1974. doi:10.1016/0370-2693(74) 90226-3.

[7] Christoph Borschensky, Michael Krämer, Anna Kulesza, Michelangelo Mangano, Sanjay Padhi, Tilman Plehn, and Xavier Portell. Squark and gluino production cross sections in pp collisions at $\sqrt{\mathrm{s}}$ =13, 14, 33 and 100 tev. Eur. Phys. J., C74(12):3174, 2014. arXiv:1407.5066, doi:10.1140/epjc/s10052-014-3174-y.

[8] ATLAS Collaboration (Aaboud, Morad et al.). Delivered luminosity versus time for 2011-2017 (p-p data only) [online]. March 2018. URL: https: / /twiki . cern. ch/twiki/bin/view/ AtlasPublic/LuminosityPublicResultsRun2 [cited 26/03/2018].

[9] Lyndon Evans and Philip Bryant. LHC Machine. JINST, 3:S08001, 2008. doi:10.1088/1748-0221/3/08/S08001.

[10] ATLAS Collaboration (Aaboud, Morad et al.). The ATLAS Experiment at the CERN Large Hadron Collider. Journal of Instrumentation, 3(08):S08003, 2008. doi:10.1088/1748-0221/3/08/S08003.

[11] M. Baak, G. J. Besjes, D. Côte, A. Koutsman, J. Lorenz, and D. Short. HistFitter software framework for statistical data analysis. Eur. Phys. J., C75:153, 2015. arXiv:1410.1280, doi:10.1140/epjc/s10052-015-3327-7.

[12] Paul Jackson, Christopher Rogan, and Marco Santoni. Sparticles in motion: Analyzing compressed susy scenarios with a new method of event reconstruction. Phys. Rev. D, 95:035031, Feb 2017. doi:10.1103/PhysRevD.95.035031.

[13] ATLAS Collaboration (Aaboud, Morad et al.). Search for squarks and gluinos in final states with jets and missing transverse momentum using $36 \mathrm{fb}^{-1}$ of $\sqrt{s}=13 \mathrm{TeV} p p$ collision data with the ATLAS detector. Submitted to PRD, 2017. arXiv:1712.02332.

[14] ATLAS Collaboration (Aaboud, Morad et al.). Search for new phenomena with large jet multiplicities and missing transverse momentum using large-radius jets and flavour-tagging at ATLAS in $13 \mathrm{TeV} p p$ collisions. JHEP, 12:034, 2017. arXiv:1708.02794, doi:10.1007/JHEP12 (2017) 034.

[15] ATLAS Collaboration (Aaboud, Morad et al.). Search for supersymmetry in final states with two same-sign or three leptons and jets using $36 \mathrm{fb}^{-1}$ of $\sqrt{s}=13 \mathrm{TeV} p p$ collision data with the ATLAS detector. JHEP, 09:084, 2017. arXiv:1706.03731, doi:10.1007/JHEP09 (2017) 084. 
[16] ATLAS Collaboration (Aaboud, Morad et al.). Search for Supersymmetry in final states with missing transverse momentum and multiple $b$-jets in proton-proton collisions at $\sqrt{s}=13 \mathrm{TeV}$ with the ATLAS detector. Submitted to JHEP, 2017. arXiv:1711.01901.

[17] ATLAS Collaboration (Aaboud, Morad et al.). ATLAS SUSY Summary. URL: https : / / atlas.web. cern. ch/Atlas / GROUPS / PHYS ICS / CombinedSummaryPlots / SUSY / [cited 12/2017].

[18] ATLAS Collaboration (Aaboud, Morad et al.). Observation of a new particle in the search for the Standard Model Higgs boson with the ATLAS detector at the LHC. Phys. Lett., B716:1-29, 2012. arXiv:1207.7214, doi:10.1016/j.physletb.2012.08.020.

[19] CMS Collaboration (Chatrchyan, Serguei et al.). Observation of a new boson at a mass of $125 \mathrm{GeV}$ with the CMS experiment at the LHC. Phys. Lett., B716:30-61, 2012. arXiv:1207. 7235, doi:10.1016/j.physletb.2012.08.021.

[20] ATLAS Collaboration (Aaboud, Morad et al.). Search for top-squark pair production in final states with one lepton, jets, and missing transverse momentum using $36 \mathrm{fb}^{-1}$ of $\sqrt{s}=13 \mathrm{TeV}$ pp collision data with the ATLAS detector. Submitted to JHEP, 2017. arXiv:1711.11520.

[21] ATLAS Collaboration (Aaboud, Morad et al.). Search for supersymmetry in events with $b$-tagged jets and missing transverse momentum in $p p$ collisions at $\sqrt{s}=13 \mathrm{TeV}$ with the ATLAS detector. JHEP, 11:195, 2017. arXiv:1708.09266, doi:10.1007/JHEP11 (2017) 195.

[22] A. Djouadi et al. The Minimal supersymmetric standard model: Group summary report. In GDR (Groupement De Recherche) - Supersymetrie Montpellier, France, April 15-17, 1998, 1998. arXiv:hep-ph/9901246.

[23] Carola F. Berger, James S. Gainer, JoAnne L. Hewett, and Thomas G. Rizzo. Supersymmetry Without Prejudice. JHEP, 02:023, 2009. arXiv:0812.0980, doi:10.1088/1126-6708/2009/02/023.

[24] ATLAS Collaboration (Aaboud, Morad et al.). Search for electroweak production of supersymmetric particles in final states with two or three leptons at $\sqrt{s}=13 \mathrm{TeV}$ with the ATLAS detector. Submitted to EPJC, 2018. arXiv:1803.02762.

[25] ATLAS Collaboration (Aaboud, Morad et al.). Search for long-lived charginos based on a disappearing-track signature in $p p$ collisions at $\sqrt{s}=13 \mathrm{TeV}$ with the ATLAS detector. Submitted to JHEP, 2017. arXiv:1712.02118.

[26] ATLAS Collaboration (Aaboud, Morad et al.). Search for new phenomena in a lepton plus high jet multiplicity final state with the ATLAS experiment using $\sqrt{s}=13 \mathrm{TeV}$ proton-proton collision data. JHEP, 09:088, 2017. arXiv:1704.08493, doi:10.1007/JHEP 09 (2017) 088. 